\title{
BMJ Open Protocol for the HAPPY Hearts study: cardiovascular screening for the early detection of future adverse cardiovascular outcomes in middle-aged and older women: a prospective, observational cohort study
}

Kevin F Boreskie, ${ }^{1,2}$ D Scott Kehler, ${ }^{1,2}$ Eduardo C Costa, ${ }^{2,3}$ Pedro C Cortez, ${ }^{2,4}$ Ivan Berkowitz, ${ }^{2}$ Naomi C Hamm, ${ }^{1,2}$ Teri L Moffatt, ${ }^{1,2}$ Andrew N Stammers, ${ }^{1,2}$ Dustin E Kimber, ${ }^{1,2}$ Brett M Hiebert, ${ }^{5}$ David E Kent, ${ }^{1,2}$ Denise E Cornish, ${ }^{1,2}$ Heather Blewett, ${ }^{6,7}$ Thang Nguyen, ${ }^{8}$ Rakesh C Arora, ${ }^{2,9}$ Shaelyn M Strachan, ${ }^{1}$ Brittany N Semenchuk, ${ }^{1}$ Jacqueline L Hay, ${ }^{1,2}$ Jay N Cohn, ${ }^{10}$ Todd A Duhamel ${ }^{1,2}$

To cite: Boreskie KF, Kehler DS, Costa EC, et al. Protocol for the HAPPY Hearts study: cardiovascular screening for the early detection of future adverse cardiovascular outcomes in middle-aged and older women: a prospective, observational cohort study. BMJ Open 2017;7:e018249. doi:10.1136/ bmjopen-2017-018249

- Prepublication history for this paper is available online. To view these files, please visit the journal online (http://dx.doi. org/10.1136/bmjopen-2017018249).

Received 16 June 2017

Revised 4 September 2017

Accepted 22 September 2017

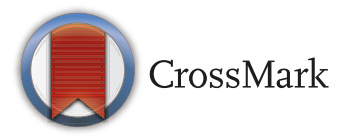

For numbered affiliations see end of article.

Correspondence to Dr Todd A Duhamel; tduhamel@sbrc.ca

\section{ABSTRACT}

Introduction Efforts to identify individuals at a higher risk for adverse cardiovascular outcomes focus on traditional risk factors, such as age, sex, smoking status, blood pressure and and cholesterol; however, this approach does not directly assess cardiovascular function and may underestimate the risk of experiencing adverse cardiovascular outcomes in women. This prospective, observational cohort study will examine the ability of the Heart Attack Prevention Program for You (HAPPY) Hearts screening protocol, a series of non-invasive procedures to identify middle-aged and older women who are at an elevated risk for experiencing an adverse cardiovascular event in the 5-year period after screening. The predictive value of the HAPPY Hearts protocol will also be compared with the Framingham Risk Score to determine the sensitivity for estimating risk for an adverse cardiovascular outcome.

Methods and analysis One thousand women 55 years of age or older will be recruited to be screened by the HAPPY Hearts protocol. This involves the cardiovascular assessment of resting blood pressure, blood pressure response to $3 \mathrm{~min}$ of moderate intensity exercise and large and small arterial elasticity. The participants will be classified into risk categories based on these measures. The incidence of the following adverse cardiovascular outcomes will be assessed in the 5-year period after screening in both groups: ischaemic heart disease, acute myocardial infarction, stroke, percutaneous coronary intervention, coronary bypass surgery, congestive heart failure and new hypertension.

Ethics and dissemination Information gathered in this research will be published in peer-reviewed journals and presented in a programme evaluation report to inform Manitoba Health and key stakeholders about the outcomes of the study. The University of Manitoba Health Research Ethics Board has approved the study protocol V.2.0, dated 29 September 2014 (H2014:224).

\section{Strengths and limitations of this study}

- The Heart Attack Prevention Program for You (HAPPY) Hearts screening protocol involves a battery of tests all involving aspects of cardiovascular disease risk. Having an array of tests will allow for the use of statistical analyses to assess different combinations of these tests in an attempt to improve the sensitivity and specificity of the HAPPY Hearts screening protocol.

- This study design utilises a convenience sample recruitment strategy, which comes with the inherent bias that the sample is unlikely to be representative of the overall population. The participants of this study, having actively chosen to participate in this study, are likely health consumers.

- As the Framingham Risk Score is considered standard of care, if participants have a primary healthcare provider, the natural history of their cardiovascular disease risk may be influenced by lipid lowering therapy, antiplatelets and other therapies meant to improve cardiovascular health.

Trial registration number NCT02863211.

\section{INTRODUCTION}

Every $7 \mathrm{~min}$ in Canada, there is a death due to heart disease or stroke. ${ }^{1}$ Up to $80 \%$ of cardiovascular disease (CVD) could be prevented by identifying and controlling for modifiable biological risk factors such as hypertension, high cholesterol and diabetes, and behavioural risk factors such as physical inactivity, an unhealthy diet, and smoking. ${ }^{2}$ 
Efforts to identify individuals at a higher risk for adverse cardiovascular events, such as the Framingham Disease Risk (FDR) score, ${ }^{3}$ focus on traditional risk factors, including age, sex, smoking status, blood pressure and cholesterol. ${ }^{4}$ While this approach does estimate 10-year risk for myocardial infarction (MI), ${ }^{3}$ it remains suboptimal. ${ }^{5-7}$ In fact, a previous study suggests that more than $80 \%$ of females evaluated with the FDR were identified as being at low risk or not qualifying for medications known to lower cardiovascular risk before experiencing a MI. ${ }^{7}$ Therefore, these data warrant the use of cardiovascular screening approaches that are more accurate in identifying the risk of adverse cardiovascular outcomes.

There is a common perception that CVD is a 'man's disease'; however, women tend to develop heart disease approximately $10-15$ years later than men, with a prevalence similar between sexes after $\geq 65$ years of age. ${ }^{8}$ Heart disease is the leading cause of death in women worldwide. ${ }^{9}$ Women are often under-represented in cardiovascular clinical trials, which is an issue because their clinical presentation and prognosis of CVD differs from that of men. ${ }^{10}$ In fact, women who suffer an acute MI are more likely to die, have an increased risk of repeat MI, develop heart failure or consequentially suffer sudden cardiac death as compared with men. ${ }^{11}$ Although improvements in CVD management have led to decreased mortality rates, more women than men have died from causes related to CVD since $1980 .{ }^{11}$ It is also important to highlight that traditional risk factors assessed by FDR score underestimate the risk of experiencing an adverse cardiovascular event in women as compared with men. ${ }^{12}$ Thus, it appears that there is a need to refine existing tools or develop innovative screening tools to better detect early stages of CVD risk among women.

The Heart Attack Prevention Program for You (HAPPY) Hearts is a cardiovascular screening programme designed to screen for cardiovascular risk in women. It was adapted from the four-test Rasmussen cardiovascular screening programme, ${ }^{13}$ which was developed by Cohn and colleagues at the Center for Cardiovascular Disease Prevention, University of Minnesota. The fourtest screening approach uses non-invasive procedures to measure structural and functional changes in the large and small arteries, including elasticity of artery walls and blood pressure at rest and in response to 3 min of moderate intensity exercise. Each of the four components of the Rasmussen Disease Score (RDS) are scored from 0 to 2 $(0=$ normal; $1=$ borderline; $2=$ abnormal $)$ with a total RDS ranging from 0 to 8 . Each participant is categorised based on risk (ie, $0-2=$ normal; $3-8=$ abnormal). These non-invasive procedures aim to detect early stages of CVD for individual asymptomatic patients. However, these tests are not yet available in the primary care setting. Moreover, it is not known if this screening approach or other protocols are feasible and have sufficient predictive value within the context of the Canadian healthcare system, where health services are generally delivered by the private sector but funded by payments from provincial health insurance.
A more comprehensive version of the RDS, the 10-test RDS, uses a number of other cardiovascular assessments in addition to the four-test approach. While a publication has shown that the 10-test RDS is better than the FDR at identifying individuals at risk for experiencing adverse cardiovascular events within $3-5$ years, ${ }^{13}$ it is unknown whether the four-test RDS is a better predictor of future cardiac events than the FDR in women. It is also unknown if the four-test screening protocol can predict future adverse cardiovascular events in women. Additionally, we will add a total of eight non-invasive tests and questionnaires to the four-test protocol in order to determine if we can improve the sensitivity of the screening protocol. We will refer to the combination of the four-test protocol plus this more extensive screening as the HAPPY Hearts protocol.

The HAPPY Hearts screening protocol is being tested specifically on women as they are often under-represented in cardiovascular clinical trials ${ }^{10}$ and their risk is underestimated using current screening methods. ${ }^{12}$ Also, arterial stiffness has been shown to have a much stronger association with mortality in women as compared with men. ${ }^{14}$ While future phases of the HAPPY Hearts research study also include men, an all-women cohort was established as a proof of concept for the screening model for these reasons.

This prospective, observational study aims to: (1) determine if the HAPPY Hearts cardiovascular screening protocol identifies women 55 years of age and older who are at an elevated risk for experiencing adverse cardiovascular outcomes within a 5-year period after screening and (2) compare the sensitivity and specificity value of the HAPPY Hearts protocol and the FDR for predicting future adverse cardiovascular outcomes in women. We hypothesise that the four-test RDS will provide better diagnostic accuracy in identifying middle-aged and older women at an elevated risk for experiencing adverse cardiovascular outcomes in the 5-year period postscreening, as compared with the FDR, and that the additional measures as part of the overall HAPPY Hearts protocol will further increase this diagnostic accuracy.

\section{METHODS AND ANALYSIS \\ Study design}

The Standard Protocol Items: Recommendations for Intervention Trials ${ }^{15}$ and the Strengthening the Reporting of Observational Studies in Epidemiology ${ }^{16}$ statements were followed in the development of this manuscript. This study is using a prospective, observational cohort design to determine if the HAPPY Hearts protocol identifies middle-aged and older women who are at a higher risk for experiencing adverse cardiovascular outcomes in the 5-year period after screening (figure 1).

\section{Study setting}

The HAPPY Hearts study will be conducted in Winnipeg, Manitoba, Canada, at the St.Boniface Hospital. Study 


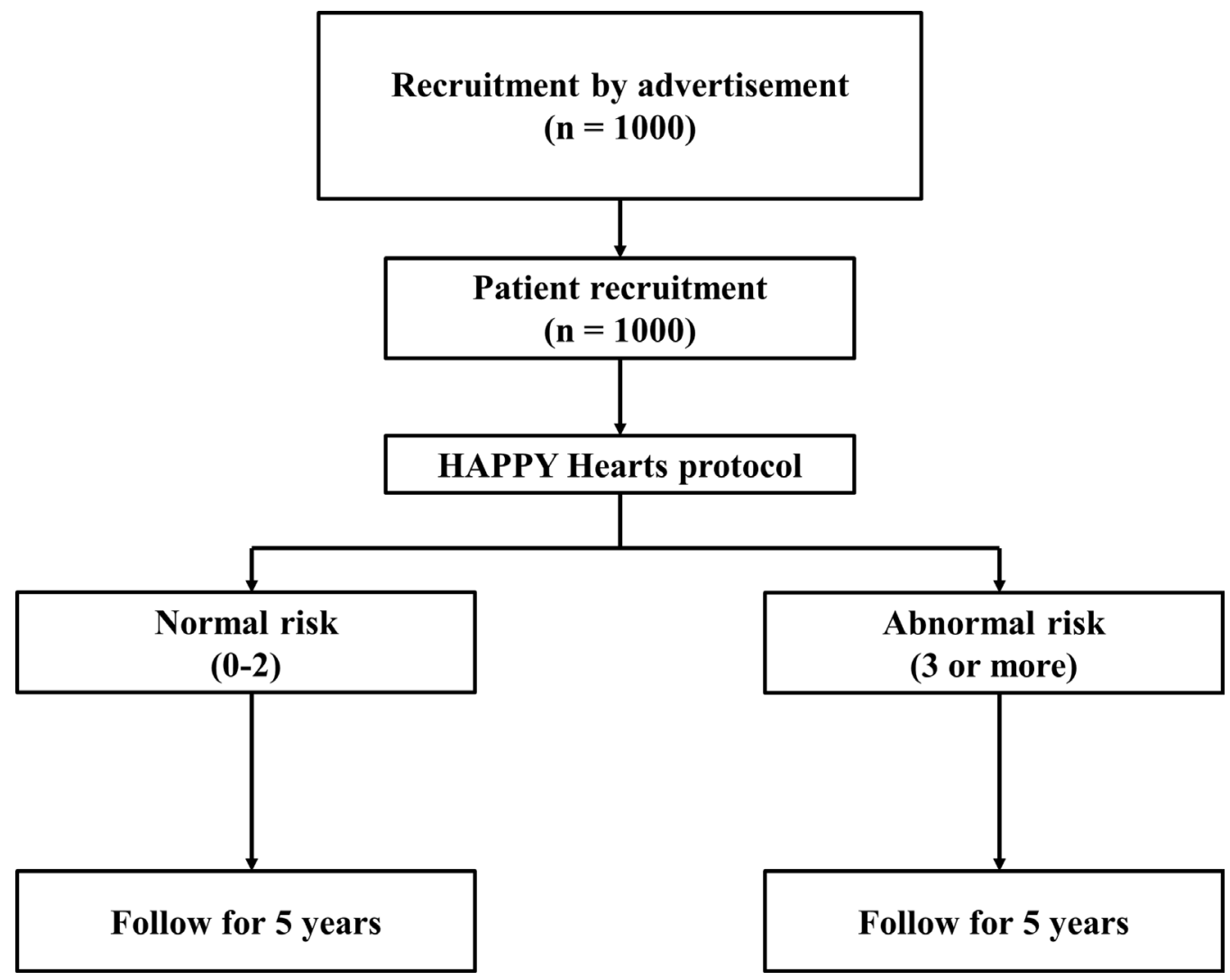

Figure 1 HAPPY Hearts flow diagram. Participant flow through the HAPPY Hearts protocol. HAPPY, Heart Attack Prevention Program for You.

recruitment was initiated in August 2015 and is expected to be completed in April 2017.

\section{Participants and recruitment}

One thousand female participants 55 years of age or older will be recruited through a convenience sample method. We used radio interviews on popular local stations as well as presentations at community events related to cardiovascular health to advertise the study to potential participants. Poster advertisements have also been placed in community centres. Individuals interested in participating were instructed to contact the research coordinator by email or telephone. Box 1 identifies the inclusion and exclusion criteria of the study. Those with hypertension were invited to participate in the study if their hypertension was being medically managed. Due to their risk profile in regard to performing physical activity, we did not include participants with hypertension that was not being medically managed. If eligible, participants were then invited to come to the I.H. Asper Clinical Research Institute, where they were given both written and verbal information describing the nature of the study. Written informed consent was obtained before enrolment. Figure 1 indicates the flow chart of the study. Table 1 identifies the adverse cardiovascular outcomes that will be included as the primary outcomes of the study. Hypertension will only be used as an outcome for those with no prior diagnosis of hypertension.

\section{The HAPPY Hearts protocol}

After patient enrolment and consent, the HAPPY Hearts protocol required participants to attend one appointment, which was scheduled for approximately $90 \mathrm{~min}$. The HAPPY Hearts protocol involves the four-test RDS protocol, which consists of four measurements (table 2): (1) resting blood pressure, (2) systolic blood pressure response following $3 \mathrm{~min}$ of moderate intensity exercise, (3) large artery elasticity assessment and (4) small artery elasticity assessment. For these measurements, the HD/

\section{Box 1 Inclusion and exclusion criteria for the}

Heart Attack Prevention Program for You (HAPPY) hearts prospective, observational cohort study

Inclusion criteria

- Women aged 55 years and older

- Possess a Manitoba Personal Health Information Number

Exclusion criteria

Previous hospitalisation for:

> ischaemic heart disease

- acute myocardial infarction

- stroke

- percutaneous coronary intervention

- coronary artery bypass surgery

- congestive heart failure

peripheral artery disease.

Previous diagnosis of hypertension, which is not medically managed. 
Table 1 Primary outcomes-adverse cardiovascular outcomes

\begin{tabular}{|c|c|}
\hline Adverse cardiovascular outcomes & Example ICD codes \\
\hline Ischaemic heart disease & $\begin{array}{l}\text { ICD-9-CM codes } 410-414 \text { or ICD-10-CA codes I20-I22, } \\
\text { I24, I25 }\end{array}$ \\
\hline Stroke & $\begin{array}{l}\text { ICD-9-CM codes } 431,434,436 \text { or ICD-10-CA codes } 161 \text {, } \\
\text { I63, } 164\end{array}$ \\
\hline Coronary artery bypass surgery & ICD-9-CM codes 36.10-36.14 or CCI code 1.IJ.76 \\
\hline $\begin{array}{l}\text { Hypertension (n.b. this outcome measure will only be used for those } \\
\text { participants with no prior diagnosis of hypertension) }\end{array}$ & $\begin{array}{l}\text { ICD-9-CM codes } 401-405 \text { or ICD-10-CA codes I10-I13, } \\
\text { I15 }\end{array}$ \\
\hline Prescription of cardiac and other medications & ATC codes \\
\hline
\end{tabular}

ICD-9-CM, International Classification of Diseases, 9th revision, clinical modification; ICD-10-CA, International Classification of Diseases, 10th revision, Canada.

PulseWave CR-2000 Research CardioVascular Profiling System (CV profiler) was used. This device is indicated for use in determining cardiovascular parameters in human subjects for research purposes only. A previous study indicated that intravisit and intervisit measures differed by less than $3 \%$ and $4 \%$, respectively, for this device. ${ }^{17}$ Research staff were trained in the use of the HD/PulseWave CR-2000 Research CardioVascular Profiling System and performed all of the testing. Below each of the measures involved in the HAPPY Hearts protocol are described. A battery of additional validated cardiovascular related testing will be conducted in addition to the previously mentioned tests. Exploratory statistical analyses (described in the Statistical

Table 2 Framingham Disease Risk Score and HAPPY Hearts protocol comparison

\begin{tabular}{|c|c|}
\hline $\begin{array}{l}\text { Framingham disease } \\
\text { risk score }\end{array}$ & HAPPY hearts protocol \\
\hline Sex & Resting systolic blood pressure \\
\hline Age & $\begin{array}{l}\text { Systolic blood pressure response to } \\
\text { exercise }\end{array}$ \\
\hline $\begin{array}{l}\text { Systolic blood } \\
\text { pressure }\end{array}$ & Large artery elasticity \\
\hline $\begin{array}{l}\text { Treatment for } \\
\text { hypertension }\end{array}$ & Small artery elasticity \\
\hline Smoking status & 6 min walking test \\
\hline Presence of diabetes & Fried frailty score \\
\hline $\begin{array}{l}\text { High-density } \\
\text { lipoprotein }\end{array}$ & CANHEART Health Index \\
\hline Total cholesterol & Additional questionnaires \\
\hline $\begin{array}{l}\text { Classification (10-year } \\
\text { risk \%) }\end{array}$ & Classification (score out of 8 ) \\
\hline Low risk $(<10 \%)$ & Normal risk (0-2) \\
\hline $\begin{array}{l}\text { Intermediate risk } \\
(\geq 10 \% \text { and }<20 \%)\end{array}$ & $\begin{array}{l}\text { Moderate to high (abnormal) risk } \\
(3-8)\end{array}$ \\
\hline
\end{tabular}

CANHEART, Cardiovascular Health in Ambulatory Care Research Team; HAPPY, Heart Attack Prevention Program for You.
Analysis section) will examine different combinations of these test results integrated with the resting blood pressure, exercise blood pressure response and arterial elasticity results in an attempt to improve the sensitivity and specificity of the HAPPY Hearts screening protocol.

Test 1: resting blood pressure

A blood pressure cuff attached to the cardiovascular profiler is placed on the participant's arm while they are in a supine position. A resting blood pressure of less than $120 / 80 \mathrm{~mm} \mathrm{Hg}$ is considered normal (0 RDS points), $120-139 / 80-89 \mathrm{~mm} \mathrm{Hg}$ is considered prehypertensive (1 RDS point) with the potential for the development of high blood pressure (hypertension). Blood pressure greater than 140/90 $\mathrm{mm} \mathrm{Hg}$ is considered abnormal for adults (2 RDS points). ${ }^{13}$ Participants with resting blood pressure measures above $160 \mathrm{~mm} \mathrm{Hg}$ systolic or $105 \mathrm{~mm} \mathrm{Hg}$ diastolic have their blood pressure remeasured following $5 \mathrm{~min}$ of rest in a supine position. If the blood pressure still exceeded these cut-offs, research assistants contacted a healthcare provider to provide medical clearance before the participant performed physical activity. All participants with resting blood pressure measures in the abnormal range, described above, received a recommendation to speak to their primary healthcare provider regarding their blood pressure.

\section{Test 2: systolic blood pressure response to exercise}

The magnitude of rise in blood pressure during exercise may be an indication of early risk for developing hypertension, even if the participant's resting blood pressure is normal. ${ }^{18}$ After the resting blood pressure is assessed, the participant is asked to perform $3 \mathrm{~min}$ of moderate exercise on a two-step stool according to the Dundee Step Test ${ }^{19}$ or on a treadmill for those who are unable to perform the step test. Both of these exercise procedures are performed at a five metabolic equivalent workload. The participant then has their blood pressure measured once again in a supine position immediately following the exercise protocol (within $30 \mathrm{~s}$ ). A rise in systolic blood pressure of under $30 \mathrm{~mm} \mathrm{Hg}$ and less than $169 \mathrm{~mm} \mathrm{Hg}$ 
absolute blood pressure is considered normal (0 RDS points), a rise of $30-39 \mathrm{~mm} \mathrm{Hg}$ and an absolute resting blood pressure of $170-179 \mathrm{~mm} \mathrm{Hg}$ is considered borderline (1 RDS point), and a rise of $40 \mathrm{~mm} \mathrm{Hg}$ or more and an absolute resting blood pressure of $180 \mathrm{~mm} \mathrm{Hg}$ or high is considered abnormal (2 RDS points) ${ }^{13}$ All participants with exercise blood pressure responses in the abnormal range received a recommendation to speak with their primary healthcare provider regarding their blood pressure.

\section{Tests 3 and 4: large and small artery elasticity}

To measure artery elasticity, a wrist stabiliser is placed on the participant's wrist while they are in a supine position. A piezoelectric transducer, or pulse wave sensor, is then placed on the location of strongest pulsation of the radial artery adjacent to the styloid process. The CV Profiler assesses the diastolic decay and waveform transmitted to it through the sensor. Based on the modified Windkessel model, the instrument can then determine small artery and large artery elasticity. Scoring of normal, borderline and abnormal results for large and small arterial elasticity are based on age and sex cut-offs. ${ }^{13}$ For females under 65 years, a large artery elasticity of $10 \mathrm{~mL} / \mathrm{mm} \mathrm{Hg} \times 10$ or greater would be normal (0 RDS points), a value from 9 to $9.9 \mathrm{~mL} / \mathrm{mm} \mathrm{Hg} \times 10$ would be considered borderline ( $1 \mathrm{RDS}$ point) and any value below $9 \mathrm{~mL} / \mathrm{mm} \mathrm{Hg} \times 10$ is considered abnormal (2 RDS points). The small artery elasticity cut-offs for this age group are $4 \mathrm{~mL} / \mathrm{mm} \mathrm{Hg}$ $\times 100$ and higher for normal (0 RDS points), $3.5-3.9 \mathrm{~mL}$ / $\mathrm{mm} \mathrm{Hg} \times 100$ for borderline (1 RDS point) and under $3.5 \mathrm{~mL} / \mathrm{mm} \mathrm{Hg} \times 100$ for abnormal (2 RDS points). Females over the age of 65 years have lower cut-offs for both large and small artery elasticity compared with the younger age category described above. Normal large artery elasticity values are $9 \mathrm{~mL} / \mathrm{mm} \mathrm{Hg} \times 10$ or higher (0 RDS points), borderline values are $8-8.9 \mathrm{~mL} / \mathrm{mm} \mathrm{Hg}$ $\times 10$ (1 RDS point) and abnormal values are under $8 \mathrm{~mL} /$ $\mathrm{mm} \mathrm{Hg} \times 10$ (2 RDS points). Normal small artery elasticity values are $3 \mathrm{~mL} / \mathrm{mm} \mathrm{Hg} \times 100$ or higher (0 RDS points), borderline values are $2.5-2.9 \mathrm{~mL} / \mathrm{mm} \mathrm{Hg} \times 100$ (1 RDS point) and abnormal values are under $2.5 \mathrm{~mL} / \mathrm{mm} \mathrm{Hg}$ $\times 100$ (2 RDS points).

\section{Scoring the four-test RDS protocol}

Each of the tests in the four-test RDS are scored as 0 for normal, 1 for borderline abnormal and 2 for abnormal. ${ }^{13}$ Total scores range from 0 to 8 and are used to stratify individuals into one of two risk groups: $0-2=$ normal risk and $\geq 3=$ moderate to high (abnormal) risk. These cut-off values have been determined through the analysis of data collected from patients of Cohn Prevention Centre licensors.

\section{Assessment of the Framingham risk score}

FDR is determined based on the calculations described by D'Agostino et al. ${ }^{3}$ Information required for this calculation is acquired from a participant questionnaire, fasting blood sampling of high density lipoprotein and total cholesterol and the resting blood pressure reading from the CV Profiler (table 2). Participants are asked to fast for the 12 hours prior to the blood draw at their appointment. Approximately $10 \mathrm{~mL}$ of blood is collected by a phlebotomist. The samples are then centrifuged so that the plasma can be separated and analysed for total cholesterol, high-density lipoprotein, low-density lipoprotein, triglycerides and fasting glucose. These variables are analysed using the cut-off values in the 2012 Canadian Cardiovascular Society/Canadian guidelines for the diagnosis and treatment of dyslipidaemia and prevention of CVD in the adult. ${ }^{20}$ Following analysis, any participants with values in the abnormal range were recommended to speak to their primary healthcare provider regarding those values.

\section{Assessment of the 2013 American College of Cardiology (ACC)/American Heart Association (AHA) risk score}

In 2013, the ACC and the AHA created a joint Guideline on the Assessment of Cardiovascular Risk task force to develop an updated multivariable risk equation for the prediction of 10-year atherosclerotic CVD risk in non-Hispanic men and women from 40 to 79 years with no prior history of atherosclerotic CVD. This updated risk calculator, as described by Goff $e t a l,{ }^{21}$ was created by pooling data from prior analyses and includes the same factors as the FRS with the addition of a race option between other and African-American. As this risk score includes the same factors as the FDR, we are able to acquire the necessary information to calculate this score in the same methods. This score will also be compared with the HAPPY Hearts protocol for its ability to identify individuals at risk for experiencing an adverse cardiovascular event within 3-5 years.

\section{Exploratory testing}

The following tests will be added to the four-test RDS in an attempt to improve the sensitivity and specificity of the overall HAPPY Hearts screening protocol.

\section{Questionnaires}

Participants are instructed to fill out a series of questionnaires:

1. Physical activity behaviour is assessed using the International Physical Activity Questionnaire (IPAQ) short version. ${ }^{22}$ This questionnaire measures the amount and intensity of physical activity completed by an individual. Physical activity has been associated with a reduction in CVD incidence and mortality. ${ }^{23}$ The IPAQ has been used to improve CVD risk scores in previous literature. ${ }^{24}$

2. The Cardiovascular Health in Ambulatory Care Research Team (CANHEART) Health Index ${ }^{25}$ is used to assess cardiovascular health behaviour and disease risk based on self-reported physical activity (from the IPAQ short version), smoking status, fruit and vegetable consumption, body mass index, as well as presence of diabetes and high blood pressure. 
3. Stage of change for health behaviours and stages of change specifically related to physical activity are assessed using the Transtheoretical Model of Change ${ }^{26}$ and the Physical Activity Stages of Change questionnaires, ${ }^{27}$ respectively. The utility of models of behaviour change have been discussed in populations with chronic disease as they can assist with the effective implementation of patient-centred interventions. ${ }^{28}$

4. Quality of life is assessed using the EuroQol Five Dimension Five Level questionnaire. ${ }^{29}$ This quality of life questionnaire has been validated for use as an outcome measure in populations with CVD. ${ }^{30}$

5. Presence and severity of depressive symptoms are assessed using the Patient Health Questionnaire-9 (PHQ-9) ${ }^{31}$ Depression has been indicated as a risk factor for CVD. ${ }^{32}$ Participants with total PHQ-9 scores in the severe depression range $(>20)$ or responses indicating thoughts of self-harm on the last question of the PHQ-9 are immediately referred to a healthcare provider at their appointment for assessment. The healthcare provider also discussed resources available for those with depressive symptoms. If no healthcare provider was immediately available, participants are escorted to the emergency room at the St. Boniface Hospital to receive assessment. Any participants with a total PHQ-9 score considered moderately severe depression (10-14) or higher receive a recommendation to speak to their primary healthcare provider.

\section{Fried frailty phenotype}

A phenotype of frailty was assessed using the Modified Fried Criteria as previously described by Fried et al. ${ }^{33}$ Briefly, the Fried score classifies participants as frail if three or more of the following criteria are met: (1) unintentional weight loss, (2) self-reported exhaustion, (3) weakness, (4) slow walking speed and (5) low physical activity. Exhaustion is assessed by the Center for Epidemiologic Studies - Depression Scale. ${ }^{34}$ Physical activity levels are assessed by the Paffenbarger Physical Activity Scale. ${ }^{35}$ Unintentional weight loss is determined via a questionnaire, while the weakness and slow walking speed are assessed using a grip strength dynamometer ${ }^{36}$ and a $5 \mathrm{~m}$ gait speed test, ${ }^{37}$ respectively. Frailty increases one's susceptibility to physiological stressors and has been shown to increase risk of cardiovascular morbidity and mortality in cohorts at risk for CVD. ${ }^{38}$

\section{6 min walking test}

Participants are instructed to walk as many lengths of a 30 $\mathrm{m}$ track as possible during the 6 min duration. Distance covered during the test is recorded. A study by Beatty et $a l^{39}$ showed the prognostic value of the 6 min walking test for predicting cardiovascular events in patients with stable coronary heart disease.

\section{Adverse cardiovascular outcomes: 5-year follow-up}

On completion of the HAPPY Hearts Protocol, each individual's Personal Health Information Number will be used to follow the study participants and to determine if they experienced an adverse cardiovascular outcome, as defined in table 1, using the Manitoba Centre for Health Policy (MCHP) Population Health Research Data Repository. International Classification of Diseases codes, as recommended by the MCHP, will be used to identify the outcomes listed in table 1 . This linkage will be done at Manitoba Health, which will provide MCHP with the correctscrambled identifiers needed to follow-up all participants. MCHP data will be accessed through a number of different databases listed in table 3. Hospital separations abstracts, medical claims and the Drug Program Information Network will be examined to provide information on hospitalisations for cardiac diseases, cardiac procedures performed, cardiac-related and other medications used and diagnoses with cardiac diseases. This information will help examine the diagnostic accuracy of the fourtest RDS, the 2013 ACC/AHA risk score and the overall HAPPY Hearts protocol versus the FDR. Vital statistics will also provide information on cause of death to allow for mortality rate tracking. This administrative health data will be used to compare adverse cardiovascular events with the HAPPY Hearts protocol results to determine if this cardiovascular screening approach predicted which individuals were at moderate to high risk of an adverse cardiovascular outcome over the 5-year period after screening. Our rationale to access all follow-up health information from the Repository at the MCHP is to obtain the most accurate information on healthcare service utilisation,

Table 3 Manitoba Centre for Health Policy data acquisition

\begin{tabular}{|c|c|c|}
\hline Database & Data fields/variables & Rationale \\
\hline Hospital Separations Abstracts & $\begin{array}{l}\text { Hospitalisation for cardiac diseases } \\
\text { and cardiac procedures performed }\end{array}$ & $\begin{array}{l}\text { Capture adverse cardiac events to test } \\
\text { diagnostic accuracy of the Rasmussen } \\
\text { Disease Score versus the Framingham } \\
\text { Disease Risk Calculator. }\end{array}$ \\
\hline Medical claims (Physician billings) & $\begin{array}{l}\text { Diagnoses with cardiac diseases and } \\
\text { procedures performed }\end{array}$ & Track adverse cardiovascular events \\
\hline Other: vital statistics & Cause of death & Track mortality rates \\
\hline Drug program information network & Cardiac and other medications used & $\begin{array}{l}\text { Track the purchases of cardiovascular } \\
\text { medications }\end{array}$ \\
\hline
\end{tabular}


adverse cardiovascular events, demographics and so on. This method is much simpler, more complete and less invasive than repeated contact with participants.

\section{Data management}

An electronic database will be used for organisation of clinical data and data analyses, which will be under the security of the St. Boniface General Hospital network. Hard copy data that contain participant identifiers will be filed in a lockable filing cabinet at the St. Boniface Hospital Albrechtsen Research Centre under the responsibility of the principal investigator and research staff. Blood samples are stored in a locked freezer in the I.H. Asper Clinical Research Institute. Only persons identified as research staff will have access to the blood samples. The St. Boniface Hospital Albrechtsen Research Centre and the I.H. Asper Clinical Research Institute are both under surveillance 24 hours a day and have at least one security guard on duty at all times. Patient identifiers will be removed during data analysis, and all medical records that contain participant identities are treated as confidential in accordance with the Personal Health Information Act of Manitoba. All study documents related to the participants will only include an assigned participant code. Blood samples will be coded with a unique participant ID number in the same way. Only research staff will have access to linkable information and will be kept confidential by law. The follow-up analyses to be conducted at MCHP will be done in accordance with MCHP's extensive security and privacy policies. ${ }^{40}$ The maximum length of time that the project data would be required is until 2027, and a request for future data will be submitted as needed. After the completion of the study, research data will be kept for a maximum of 10 years and then destroyed.

\section{Statistical analysis}

Rates of expected adverse health outcomes in the HAPPY Hearts cohort were calculated using age and sex stratified Manitoban data from two published reports, namely Sex Differences in Health Status, Health Care Use, and Quality of Care: A Population-Based Analysis for Manitoba's Regional Health Authorities ${ }^{41}$ and age stratified Canadian data from the Chronic Disease and Injury Indicator Framework. ${ }^{42}$ Information from these documents were used to calculate incident rates of the adverse health outcomes listed in table 1 based on the expected age of participants within the sample population. In some cases, incident rates were not directly available in the reports and, therefore, were estimated using reported prevalence data. Based on this calculation, we predict a total of 187-241 adverse health outcomes in the HAPPY Hearts cohort. This estimate is based on the following rates of adverse health outcomes: 14-29 incidents of ischaemic heart disease; 1-4 incidents of acute MI; 3-16 incidents of stroke; 1 incident of coronary artery bypass graft; 6 incidents of congestive heart failure; 79 new prescriptions for cardiac medications (ACE inhibitors and statins); and 83-102 new diagnoses of hypertension. Comparative predictability between the four-test RDS, overall HAPPY Hearts protocol, 2013 ACC/AHA risk score and FDR protocols will be assessed using survival analysis techniques, such as Kaplan-Meier methods or a Cox proportional hazards model, and calculating c-statistics. Model discrimination will be assessed using techniques like receiver operating characteristic curves and integrated discrimination improvement. Net Reclassification Improvement will be used to assess change in risk category for participants between the RDS, ACC/AHA risk score and FDR screening protocols. Based on work done by Harrell $e t a t^{43}$ and validated by Peduzzi et a $t^{44}$ suggesting that there should be at least 10 outcome events per variable in the model, we will conservatively be able to use 10 variables in our model. Distribution and end results may dictate analyses used. In order to compare the HAPPY Hearts screening method to the FDR and the 2013 ACC/ AHA risk score, our first analysis model will not include incident hypertension, as these screening methods do not include this outcome. We will add incident hypertension to the composite outcome in a second model. While traditional models do not include this measure, the HAPPY Hearts protocol includes measures that have been demonstrated to identify subjects at increased risk of future incidence of hypertension. ${ }^{45}$

\section{ETHICS}

Any amendments, where necessary, will be submitted for review and approval prior to implementation. Study status will be reported annually. A final study notification will be forwarded at completion of the study or in the event of early termination to the University of Manitoba Health Research Ethics Board. The tenets of Good Clinical Practice will also be followed. Subjects are free to withdraw from the study at any time without affecting their right to medical care.

\section{End of study}

The study recruitment will end when 1000 participants have been enrolled. Adverse cardiovascular event data will then be analysed 5 years after the final participant was screened using the MCHP Population Health Research Data Repository.

\section{Study oversight}

The HAPPY Hearts research team at the St. Boniface Hospital Albrechtsen Research Centre will be responsible for all facets of the study including the design, ongoing management, ethical conduct, statistical analysis and dissemination of the results.

\section{DISSEMINATION}

The anonymised information gathered from the data collection process will be summarised (eg, means $\pm \mathrm{SD}$ ), disseminated into a report and shared with the St. Boniface Hospital Foundation, investigators of the study and stakeholders involved in this study. The information will be presented at 
conferences or published in peer-reviewed journals. Neither participants' names nor contact information will appear in any publications stemming from this research.

\section{STUDY STATUS}

Recruitment for the HAPPY Hearts study began in August 2015. As of 25 May 2016, 500 participants have been recruited to the HAPPY Hearts study. Recruitment completion is expected to be in October 2017.

\section{Author affiliations}

${ }^{1}$ Faculty of Kinesiology and Recreation Management, Health, Leisure \& Human

Performance Research Institute, University of Manitoba, Winnipeg, Manitoba,

Canada

${ }^{2}$ Institute of Cardiovascular Sciences, St. Boniface Hospital Albrechtsen Research

Centre, Winnipeg, Manitoba, Canada

${ }^{3}$ Department of Physical Education, Federal University of Rio Grande do Norte, Natal, Rio Grande do Norte, Brazil

${ }^{4}$ Federal University of Para, Para, Brazil

${ }^{5}$ Department of Cardiac Sciences Program, Winnipeg Regional Health Authority, Winnipeg, Manitoba, Canada

${ }^{6}$ Agriculture and Agri-Food Canada, Government of Canada, Winnipeg, Manitoba, Canada

${ }^{7}$ Department of Human Nutritional Sciences, University of Manitoba, Winnipeg, Manitoba, Canada

${ }^{8}$ Section of Cardiology, University of Manitoba, Max Rady College of Medicine, Winnipeg, Manitoba, Canada

${ }^{9}$ Department of Surgery, University of Manitoba, Max Rady College of Medicine, Winnipeg, Manitoba, Canada

${ }^{10}$ Rasmussen Center for Cardiovascular Disease Prevention, University of Minnesota Medical School, Minneapolis, Minnesota, USA

Acknowledgements The authors would like to acknowledge the infrastructure support provided by the St. Boniface Hospital Albrechtsen Research Centre and the I.H. Asper Clinical Research Institute. Operating funds to support this research was generously provided by the St. Boniface Hospital Research Foundation, the Canadian Pacific Railway and the Sir Thomas Cropo Foundation Inc. The authors would also like to thank Terry MacLeod on CBC Radio One, Chuck LaFleche and Greg Mackling on the CJ0B Health Report, CKJS 810AM, the Filipino Express News Magazine and the Women's Heart Health Forum at the Westminster United Church for their assistance in recruiting study participants.

Contributors TAD, DSK and IB were involved in conception and design of research. TAD and IB secured funding for the project. DSK, TAD and RCA obtained ethics approval. ECC prepared the figures. KFB, ECC, PCC, DSK and TAD drafted the manuscript. All authors edited and revised the manuscript. All authors approved the final version of the manuscript. KFB, ECC, PCC, DSK, NCH, TLM, ANS, DEK, JLH, BNS and DEC assisted with data collection and analysis. BH provided statistical support. KFB and DEK provided research administration support. HB assisted with blood sample analysis.

Funding The study is being financially supported by an operating grant from the St. Boniface Hospital Foundation. KFB is supported by a Manitoba Graduate Scholarship and the Graduate Enhancement of Tri-Council Stipends program. ECC is supported by the National Council for Scientific and Technological Development (CNPq; process 201422/2015-8). PCC is supported by the National Council for Scientific and Technological Development. DSK is supported by a Canadian Institutes of Health Research Frederick Banting and Charles Best Canada Graduate Scholarship. ANS is supported by a CIHR Frederick Banting and Charles Best Canada Graduate Scholarship, the Ruth Asper Scholarship in Physical Education, Kinesiology and Recreation and a University of Manitoba Jack MacDonnell Scholarship for Research in Aging. JLH and NCH are supported by the University of Manitoba Graduate Fellowship program.

Competing interests The 4-Test Rasmussen cardiovascular screening program protocol is protected by intellectual property owned by the University of Minnesota and licensed to CVC-HEARTSAVERS LLC. St. Boniface General Hospital has a service agreement to utilize the methodology.
Ethics approval The study protocol V.3.0 has been reviewed by the University of Manitoba Health Research Ethics Board and has been approved V.3.0 (H2014:224) on August 15, 2016.

Provenance and peer review Not commissioned; externally peer reviewed.

Data sharing statement Data for the HAPPY Hearts study can be made available upon request. Interested researchers should contact Dr TAD at tduhamel@sbrc.ca.

Open Access This is an Open Access article distributed in accordance with the Creative Commons Attribution Non Commercial (CC BY-NC 4.0) license, which permits others to distribute, remix, adapt, build upon this work non-commercially, and license their derivative works on different terms, provided the original work is properly cited and the use is non-commercial. See: http://creativecommons.org/ licenses/by-nc/4.0/

(C) Article author(s) (or their employer(s) unless otherwise stated in the text of the article) 2017. All rights reserved. No commercial use is permitted unless otherwise expressly granted.

\section{REFERENCES}

1. Government of Canada SC. CANSIM - Canadian socioeconomic database from Statistics Canada. 2016 http://www5.statcan.gc.ca/ cansim/a01?lang=eng\&p2=1 (accessed $15 \mathrm{Jul} 2016$ ).

2. WHO / Strategy documents. WHO. http://www.who.int/ dietphysicalactivity/strategy/eb11344/en/ (accessed 15 Jul 2016).

3. D'Agostino RB, Vasan RS, Pencina MJ, et al. General cardiovascular risk profile for use in primary care: the Framingham Heart Study. Circulation 2008;117:743-53.

4. Wilson PW, D'Agostino RB, Levy D, et al. Prediction of coronary heart disease using risk factor categories. Circulation 1998;97:1837-47.

5. Akosah KO, Schaper A, Cogbill C, et al. Preventing myocardial infarction in the young adult in the first place: how do the National Cholesterol Education Panel III guidelines perform? J Am Coll Cardiol 2003:41:1475-9.

6. Yoon YE, Rivera JJ, Kwon DA, et al. National Cholesterol Education Panel III guidelines performance role in preventing myocardial infarction in a large cohort without a history of coronary artery disease: Korea Acute Myocardial Infarction Registry study. Prev Cardiol 2009;12:109-13.

7. Dib JG, Alameddine Y, Geitany R, et al. National cholesterol education panel III performance in preventing myocardial infarction in young adults. Ann Saudi Med 2008;28:22-7.

8. Women and Health. http://www.statcan.gc.ca/pub/89-503-x/ 2010001/article/11543-eng.htm\#a11 (accessed 10 May 2017).

9. WHO |Women's health. http://www.who.int/mediacentre/factsheets/ fs334/en/ (accessed 27 Jul 2016).

10. Vitale C, Rosano G, Fini M. Are elderly and women underrepresented in cardiovascular clinical trials? Implication for treatment. Wien Klin Wochenschr 2016;128:433-8.

11. Go AS, Mozaffarian D, Roger VL, et al. Heart disease and stroke statistics-2013 update: a report from the american heart association. Circulation 2013;127:e6-245.

12. Rodondi N, Locatelli I, Aujesky D, et al. Framingham risk score and alternatives for prediction of coronary heart disease in older adults. PLoS One 2012;7:e34287.

13. Duprez DA, Florea N, Zhong W, et al. Vascular and cardiac functional and structural screening to identify risk of future morbid events: preliminary observations. J Am Soc Hypertens 2011;5:401-9.

14. Regnault V, Thomas F, Safar ME, et al. Sex difference in cardiovascular risk: role of pulse pressure amplification. J Am Coll Cardiol 2012;59:1771-7.

15 SPIRIT Statement. http://www.spirit-statement.org/ (accessed 28 Aug 2017).

16. STROBE Statement. Home. https://www.strobe-statement.org/index. php?id=strobe-home. (accessed 25 Mar 2017).

17. Zimlichman R, Shargorodsky M, Boaz M, et al. Determination of arterial compliance using blood pressure waveform analysis with the CR-2000 system: reliability, repeatability, and establishment of normal values for healthy european population--the seven european sites study (SESS). Am J Hypertens 2005;18:65-71.

18. Singh JP, Larson MG, Manolio TA, et al. Blood pressure response during treadmill testing as a risk factor for new-onset hypertension. The framingham heart study. Circulation 1999;99:1831-6.

19. Lim P, Shiels P, Anderson J, et al. Dundee step test: a simple method of measuring the blood pressure response to exercise. J Hum Hypertens 1999;13:521-6.

20. Anderson TJ, Grégoire J, Hegele RA, et al. 2012 update of the canadian cardiovascular society guidelines for the diagnosis and 
treatment of dyslipidemia for the prevention of cardiovascular disease in the adult. Can J Cardiol 2013;29:151-67.

21. Goff DC, Lloyd-Jones DM, Bennett G, et al. ACC/AHA guideline on the assessment of cardiovascular risk. Circulation 20132013;98.

22. Craig CL, Marshall AL, Sjöström M, et al. International physical activity questionnaire: 12 -country reliability and validity. Med Sci Sports Exerc 2003;35:1381-95.

23. Haennel RG, Lemire F. Physical activity to prevent cardiovascular disease. How much is enough? Can Fam Physician 2002;48:65-71.

24. Georgousopoulou EN, Panagiotakos DB, Bougatsas D, et al. Physical Activity Level Improves the Predictive Accuracy of Cardiovascular Disease Risk Score: The ATTICA Study (2002-2012). Int J Prev Med $2016 ; 7$.

25. Maclagan LC, Park J, Sanmartin C, et al. The canheat health index: a tool for monitoring the cardiovascular health of the canadian population. CMAJ 2014;186:180-7.

26. Prochaska JO, DiClemente CC. Stages and processes of self-change of smoking: toward an integrative model of change. J Consult Clin Psychol 1983;51:390-5.

27. Marcus $\mathrm{BH}$, Forsyth LH. Motivating people to be physically active. Human Kinetics. Champaign, IL, 2003.

28. Gillespie ND, Lenz TL. Implementation of a Tool to Modify Behavior in a Chronic Disease Management Program. Adv Prev Med 2011;2011:1-5.

29. Herdman M, Gudex C, Lloyd A, et al. Development and preliminary testing of the new five-level version of EQ-5D (EQ-5D-5L). Qual Life Res 2011;20:1727-36.

30. Dyer MT, Goldsmith KA, Sharples LS, et al. A review of health utilities using the EQ-5D in studies of cardiovascular disease. Health Qual Life Outcomes 2010;8:13.

31. Kroenke K, Spitzer RL, Williams JB. The PHQ-9: validity of a brief depression severity measure. J Gen Intern Med 2001;16:606-13.

32. Blumenthal JA. Depression and coronary heart disease: association and implications for treatment. Cleve Clin J Med 2008;75 Suppl 2(Suppl 2):S48-53.

33. Fried LP, Tangen CM, Walston J, et al. Frailty in older adults: evidence for a phenotype. J Gerontol A Biol Sci Med Sci 2001;56:M146-M157.

34. Radloff LS. The CES-D scale a self-report depression scale for research in the general population. Appl Psychol Meas 1977;1:385-401.

35. Nowak Z, Plewa M, Skowron M, et al. Paffenbarger physical activity questionnaire as an additional tool in clinical assessment of patients with coronary artery disease treated with angioplasty. Kardiol Pol 2010;68:32-9.

36. Leong DP, Teo KK. Predicting cardiovascular disease from handgrip strength: the potential clinical implications. Expert Rev Cardiovasc Ther 2015;13:1277-9.

37. Schoon Y, Bongers K, Van Kempen J, et al. Gait speed as a test for monitoring frailty in community-dwelling older people has the highest diagnostic value compared to step length and chair rise time. Eur $J$ Phys Rehabil Med 2014;50:693-701.

38. Afilalo J, Alexander KP, Mack MJ, et al. Frailty assessment in the cardiovascular care of older adults. J Am Coll Cardiol 2014;63:747-62.

39. Beatty AL, Schiller NB, Whooley MA. Six-minute walk test as a prognostic tool in stable coronary heart disease: data from the heart and soul study. Arch Intern Med 2012;172:1096-102.

40 University of Manitoba - Faculty of Medicine - Community Health Sciences - Manitoba Centre for Health Policy - Pledge of Privacy. http://umanitoba.ca/faculties/health_sciences/medicine/units/ community_health_sciences/departmental_units/mchp/privacy.html (accessed 11 Aug 2016)

41. University of Manitoba - Faculty of Medicine - Community Health Sciences - Manitoba Centre forHealth Policy - Data Extras for Sex Differences in Health Status, Health Care Use, and Quality ofCare. A Population-Based Analysis for Manitoba's Regional Health Authoritie. http://umanitoba.ca/faculties/health_sciences/medicine/units/chs/ departmental_units/mchp/projects/ntk-sex.html. (accessed 9 May 2017).

42. Chronic Disease and Injury Indicator Framework. Public Health Infobase - Canada.ca. http://infobase.phac-aspc.gc.ca/cdiif/. (accessed 9 May 2017).

43. Regression modelling strategies for improved prognostic prediction. Harrell - 1984 - Statistics in Medicine - Wiley Online Library. http:// onlinelibrary.wiley.com/doi/. (accessed 28 Aug 2017).

44. Peduzzi P, Concato J, Feinstein AR, et al. Importance of events per independent variable in proportional hazards regression analysis. II. Accuracy and precision of regression estimates. J Clin Epidemiol 1995;48:1503-10.

45. Keller K, Stelzer K, Ostad MA, et al. Impact of exaggerated blood pressure response in normotensive individuals on future hypertension and prognosis: Systematic review according to PRISMA guideline. Adv Med Sci 2017;62:317-29. 